\title{
Genome Resource for Two Stemphylium vesicarium Isolates Causing Stemphylium Leaf Blight of Onion in New York
}

\author{
Sandeep Sharma, Frank S. Hay, and Sarah J. Pethybridge ${ }^{\dagger}$ \\ Plant Pathology and Plant-Microbe Biology Section, School of Integrative Plant Science, Cornell \\ AgriTech at the New York State Agricultural Experiment Station, Cornell University, Geneva, NY 14456, \\ U.S.A.
}

\begin{abstract}
Stemphylium leaf blight caused by Stemphylium vesicarium was recently identified as an emerging disease and dominant in the foliar disease complex affecting onion in New York. Here, we report the genomes of two isolates of $S$. vesicarium, On16-63 and On16-391. The availability of the genomes will accelerate genomic studies of $S$. vesicarium, including population biology, sexual reproduction, and fungicide resistance. Additionally, comparative genomics with the other published genome of $S$. vesicarium causing brown spot of pear will help understand pathogen biology and underpin the development of management strategies for this disease.
\end{abstract}

\section{Genome Announcement}

Stemphylium vesicarium causes disease of a broad range of economically important plants including vegetables, herbaceous crops, and fruit trees (Falloon et al. 1987; Lamprecht et al. 1984; Rossi et al. 2005; Suheri and Price 2000). Stemphylium leaf blight (SLB) caused by S. vesicarium was first reported in the United States in Texas in 1976 (Miller et al. 1978) and was usually considered a minor component of the foliar disease complex affecting onion worldwide but has recently emerged as dominant in New York (Hay et al. 2019) and Ontario, Canada (Foster et al. 2019; Paibomesai et al. 2012). This has been attributed to the development of resistance against commonly used fungicides (Hay et al. 2019). Despite the importance of SLB on onion, genomic resources and population biology studies on $S$. vesicarium are limited. The first genome of $S$. vesicarium associated with brown spot of pear was only recently released (Gazzetti et al. 2019). To accelerate genomic studies, develop tools to understand the population biology and reproduction, and identify genes responsible for fungicide resistance, the genomes of $S$. vesicarium isolates On16-63 and On16-391, associated with SLB epidemics of onion in New York (Hay et al. 2019), were sequenced and assembled.

S. vesicarium On16-63 and On16-391 were isolated from SLB on onion from New York in 2016. The identity of the isolates was confirmed through multilocus sequencing and phylogenetic analysis (Hay et al. 2019). DNA was extracted from single-spore cultures using a modified CTAB extraction protocol (Leslie and Summerell 2006). DNA quantity was assessed with a Nanodrop ND 1000 spectrophotometer and quality with a Qubit fluorometer. Genomic

${ }^{\dagger}$ Corresponding author: S. J. Pethybridge; sjp277@ cornell.edu

The author(s) declare no conflict of interest.

Accepted for publication 9 January 2020.

(C) 2020 The American Phytopathological Society

\section{Funding}

This research was supported by the United States Department of Agriculture National Institute of Food and Agriculture Hatch project NYG-625445, managed by Cornell AgriTech, Cornell University, Geneva, New York, and Federal Capacity Fund Initiative Project 2016-17-149.

Keywords

Allium cepa, foliar disease 
Table 1. Genome features of Stemphylium vesicarium isolates On16-63 and On16-391 from Stemphylium leaf blight of onion compared with the published genome of Stemphylium vesicarium 173-1a13FI1M3 associated with brown spot of pear (Gazzetti et al. 2019)

\begin{tabular}{lccc} 
Features & On16-63 & On16-391 & $\mathbf{1 7 3 - 1 a 1 3 F I 1 M 3 ~}$ \\
Number of contigs & $1,734(>500 \mathrm{bp})$ & $2,395(>500 \mathrm{bp})$ & $1,127(>0 \mathrm{bp})$ \\
N50 (bp) & 118,769 & 70,493 & 167,978 \\
L50 (bp) & 92 & 150 & 63 \\
Largest contig (bp) & 644,906 & 572,557 & 974,405 \\
Assembly length (all contigs) & $40,827,444$ & $39,846,678$ & $38,664,579$ \\
Genome coverage & $97 \times$ & $110 \times$ & $60 \times$ \\
GC content (\%) & 50.68 & 51.04 & 50.72 \\
BUSCO completeness (\%) & 98.6 & 98.7 & 96 \\
Predicted genes & 13,064 & 12,810 & 12,309 \\
tRNAs & 380 & 366 & N/A \\
\hline
\end{tabular}

DNA (5 $\mu$ g for each isolate, On16-63 and On16-391) was submitted to the Cornell University Institute of Biotechnology Genomics Facility, Ithaca, New York, for PCR-free library preparation. Libraries were prepared with a TrueSeq Nano DNA library preparation kit, and $2 \times$ 300-bp paired-end sequencing was performed using an Illumina MiSeq. Both isolates were sequenced within a single lane. The quality of the reads was determined with FastQC $v 0.11 .8$ (Andrews 2010). Poor-quality reads with an average quality score less than 30 were trimmed and discarded with Trimmomatic version 0.36 (Bolger et al. 2014). High-quality reads were assembled with AbySS version 1.9.0 (Simpson et al. 2009). QUAST-LG (Mikheenko et al. 2018) was used to predict the size of the genomes and sequencing coverage. The completeness and quality of the assembled genomes was analyzed with Benchmarking Universal single-copy orthologs (BUSCO v 3.1.0) (Simão et al. 2015). Putative genes in the isolates were predicted with AUGUSTUS version 3.3.2 (Stanke and Morgenstern 2005), using gene sets of Fusarium graminearum. tRNAscan-SE version 1.3.1 (Lowe and Chan 2016) was used to predict the number of transfer RNAs (tRNAs) in the genomes. To identify the loci associated with the mating types and resistance against commonly used fungicides, BLAST databases for the S. vesicarium isolates On16-63 and On16-391 were created, using Geneious version 11.0.5 (Kearse et al. 2012). Nucleotide sequences of cytochrome b, hexokinase, succinate dehydrogenase (SDHB, C, D) and cyp51 from Stemphylium or closely related Alternaria species were retrieved from GenBank and searched as queries against the local BLAST database created for isolates On16-63 and On16-391. Similarly, sequences of the mating type (MAT) genes previously deposited in GenBank (AY335178.1) (Inderbitzin et al. 2005) were downloaded and searched as queries against the database created for the isolates.

A single-lane Illumina MiSeq $2 \times 300$ sequencing of the libraries produced $2 \times 8.47$ million reads and $2 \times 9.81$ million reads for $S$. vesicarium isolates On16-63 and On16-391, respectively. The genomes were assembled into 1,734 (On16-63) and 2,393 (On16-391) contigs greater than 500 base pairs with a coverage of approximately $97 \times$ and $103 \times$ (Table 1). The estimated genome sizes were $40.83 \mathrm{Mb}$ and $39.85 \mathrm{Mb}$, with a GC content of $50.68 \%$ (On16-63) and 51.04\% (On16-391). The predicted sizes of the genomes were similar to a S. vesicarium isolate from pear (Gazzetti et al. 2019). For On16-63, the N50 value was 118,769 and the largest contig was 644,906 bp. For On16-391, the N50 value was 70,493 and the largest contig was $572,557 \mathrm{bp}$. BUSCO analysis predicted the genome assemblies to be 98.7 and $98.6 \%$ complete for isolates On16-63 and On16-391, respectively. AUGUSTUS predicted 13,064 genes for the assembled genome of isolate On16-63 and 12,810 genes for isolate On16-391. Based on the results of tRNAscan-SE, 380 and 366 tRNAs were predicted in the assembled genomes of isolates On16-63 and On16-391, respectively. Furthermore, BLAST analyses of genes for fungicide resistance against the genomes successfully identified different loci associated with some commonly used fungicides. Both $S$. vesicarium isolates contained fused MAT1-1 and MAT1-2 genes in reverse orientation, a feature previously demonstrated in some Stemphylium spp. (Inderbitzin et al. 2005). Single copies of MAT1-1 and MAT1-2 were identified in both genomes.

To the best of our knowledge, this is the first report of the genome of $S$. vesicarium causing SLB of onion. These genomes will be a useful resource to enable pathogen and population biology studies and to develop molecular-based tools for fungicide resistance monitoring. 
The $S$. vesicarium isolates were deposited in the International Collection of Microorganisms from Plants (ICMP) (Auckland, New Zealand) (On16-63 = ICMP 23434; On16-391 = ICMP 23435). The whole genome shotgun projects have been deposited at DDBJ/ENA/ GenBank under accessions VHPT00000000 and VHPU00000000. Draft assemblies described herein are versions VHPT01000000 and VHPU01000000 for isolates On16-63 and On16-391, respectively. Raw sequencing reads are available in GenBank as SRA accession PRJNA549936.

\section{Acknowledgments}

We thank Q. Sun from the Computational Biology Service Unit, Cornell University, for assistance with the bioinformatics analyses.

\section{Author-Recommended Internet Resource}

Geneious version 11.0.5: https://www.geneious.com

\section{Literature Cited}

Andrews, S. 2010. FastQC: A quality control tool for high throughput sequence data. Babraham Bioinformatics, Babraham Institute, Cambridge. Published online. http://www.bioinformatics.babraham.ac.uk/projects/fastqc

Bolger, A. M., Lohse, M., and Usadel, B. 2014. Trimmomatic: A flexible trimmer for Illumina sequence data. Bioinformatics 30:2114-2120.

Falloon, P. G., Falloon, L. M., and Grogan, R. G. 1987. Etiology and epidemiology of Stemphylium leaf spot and purple spot of asparagus in California. Phytopathology 77:407-413.

Foster, J. M., Tayviah, C. S., Stricker, S. M., Gossen, B. D., and McDonald, M. R. 2019. Susceptibility to Stemphylium vesicarium of asparagus, onion, pear, and rye in Canada. Can. J. Plant Pathol. 41:228-241.

Gazzetti, K., Diaconu, E. L., Nanni, I. M., Ciriani, A., and Collina, M. 2019. Genome sequence resource for Stemphylium vesicarium, causing brown spot disease of pear. Mol. Plant-Microbe Interact. 32:935-938.

Hay, F. S., Sharma, S., Hoepting, C., Strickland, D., Luong, K., and Pethybridge, S. J. 2019. Emergence of Stemphylium leaf blight of onion in New York attributed to fungicide resistance. Plant Dis. 103:3083-3092.

Inderbitzin, P., Harkness, J., Turgeon, B. G., and Berbee, M. L. 2005. Lateral transfer of mating system in Stemphylium. Proc. Natl. Acad. Sci. U.S.A. 102:11390-11395.

Kearse, M., Moir, R., Wilson, A., Stones-Havas, S., Cheung, M., Sturrock, S., Buxton, S., Cooper, A., Markowitz, S., Duran, C., Thierer, T., Ashton, B., Meintjes, P., and Drummond, A. 2012. Geneious Basic: An integrated and extendable desktop software platform for the organization and analysis of sequence data. Bioinformatics 28:1647-1649.

Lamprecht, S. C., Baxter, A., and Thompson, A. H. 1984. Stemphylium vesicarium on Medicago spp. in South Africa. Phytophylactica 16:73-75.
Leslie, J. F., and Summerell, B. A. 2006. Nucleic acid analyses. Pages 57-77 in: The Fusarium Laboratory Manual. Blackwell Publishing, Oxford.

Lowe, T. M., and Chan, P. P. 2016. tRNAscan-SE On-line: Integrating search and context for analysis of transfer RNA genes. Nucleic Acids Res. 44 (W1): W54-W57.

Mikheenko, A., Prjibelski, A., Saveliev, V., Antipov, D., and Gurevich, A. 2018. Versatile genome assembly evaluation with QUAST-LG. Bioinformatics 34: i142-i150.

Miller, M. E., Taber, R. A., and Amador, J. M. 1978. Stemphylium blight of onion in south Texas. Plant Dis. Rep. 62:851-853.

Paibomesai, M., Celetti, M., and Tesfaendrias, M. 2012. Update on Stemphylium leaf blight in onions in Ontario. HortMatters 12:11-12.

Rossi, V., Pattori, E., Giosué, S., and Bugiani, R. 2005. Growth and sporulation of Stemphylium vesicarium, the causal agent of brown spot of pear, on herb plants of orchard lawns. Eur. J. Plant Pathol. 111:361-370.

Simão, F. A., Waterhouse, R. M., loannidis, P., Kriventseva, E. V., and Zdobnov, E. M. 2015. BUSCO: Assessing genome assembly and annotation completeness with single-copy orthologs. Bioinformatics 31:3210-3212.

Simpson, J. T., Wong, K., Jackman, S. D., Schein, J. E., Jones, S. J., and Birol, I. 2009. ABySS: A parallel assembler for short read sequence data. Genome Res. 19:1117-1123

Stanke, M., and Morgenstern, B. 2005. AUGUSTUS: A web server for gene prediction in eukaryotes that allows user-defined constraints. Nucleic Acids Res. 33 (Web Server):W465-W467.

Suheri, H., and Price, T. V. 2000. Infection of onion leaves by Alternaria porri and Stemphylium vesicarium and disease development in controlled environments. Plant Pathol. 49:375-382. 